\title{
Visual hierarchy relates to impressions of good design
}

\author{
YOKO URANO
}

Princeton University, yurano@princeton.edu

AARON KUROSU

Princeton University, akurosu@alumni.princeton.edu

GREGORY HENSELMAN-PETRUSEK

Oxford University, greg.henselman@gmail.com

\section{ALEXANDER TODOROV}

The University of Chicago, Booth School of Business, alexander.todorov@chicagobooth.edu

Here we examine an untested assumption among graphic designers that a concept called "visual hierarchy" is tied to the perception of good design. Visual hierarchy refers to the sequence in which graphic elements in a design are seen. From a design perspective, a stronger visual hierarchy means that a graphic design, such as a poster, will lead to more similar eye movements among its audience. From a psychology perspective, stronger visual hierarchy may mean that information for guiding overt attention is being more effectively communicated. The consequent cognitive ease may facilitate an aesthetic experience, thereby explaining how visual hierarchy could be linked to perceptions of good design. In an empirical test, we see that when people agree that a design is good, their eye movements are more likely to be synchronous.

CCS CONCEPTS • Human-centered computing

Additional Keywords and Phrases: Graphic design, visual hierarchy, Fréchet distance, eye tracking, judgement, attention, synchrony, scan path, social perception

\section{ACM Reference Format:}

Yoko Urano, Aaron Kurosu, Gregory Henselman-Petrusek, and Alexander Todorov. 2021. Visual Hierarchy Relates to Impressions of Good Design. In EMICS '21: ACM CHI '21 Workshop on Eye Movements as an Interface to Cognitive State, May 14, 2021, Yokohama, Japan. ACM, New York, NY, USA, 8 pages.

\section{INTRODUCTION}

Visual hierarchy in graphic design refers to the potential for graphics to guide people's attention in a prescribed sequence [3, 9]. Graphic designers will often structure a hierarchy to control the experience of their audience [12]. For example, a newspaper may use a large, emboldened font to draw people's attention to what an editor believes is most important first [9]. On the same page, additional stories may have their size and position structured to impart their relative importance [5, 9]. Graphic designers developed this notion heuristically, but

Permission to make digital or hard copies of part or all of this work for personal or classroom use is granted without fee provided that copies are not made or distributed for profit or commercial advantage and that copies bear this notice and the full citation on the first page. Copyrights for third-party components of this work must be honored. For all other uses, contact the owner/author(s).

EMICS '21, May 14, 2021, Yokohama, Japan

(c) 2021 Copyright held by the owner/author(s). 
its validity as a scientific concept remains unknown. The purpose of this paper is to test if the presence of visual hierarchy indeed relates to the perception of good design.

Theoretically speaking, the notion of visual hierarchy is consonant with two dominant theories of attention and aesthetics: the pre-motor theory of attention and the fluency theory of aesthetics. According to the premotor theory of attention, overt attention involves saccades that place regions of interest at the fovea; in contrast, exogeneous covert attention occurs independent of eye movement, although it nevertheless depends on saccade planning $[2,17]$. Overt and covert processes may work together to provide a person with information about what they are seeing and where to shift their attention. For example, when someone first looks at a web page, they are likely to look first towards the upper left [13]. This could be explained by a covert process that combines visual cues and prior experiences to form a prediction that looking towards the upper left will provide the most diagnostic information about what is being seen. The fluency theory of aesthetics posits that pleasurable sensations may come, at least in part, from efficient processing and cognitive ease [18]. Therefore, the efficiency of covert and overt processes in extracting information may engender an aesthetic response. Together, these theories situate our hypothesis that graphics with stronger visual hierarchy are more likely to be perceived as having a good design.

The potential value of examining sequences of eye movements has been pointed out in prior research [1]. However, a direct test of visual hierarchy has not been conducted - not likely due to a lack of interest, but rather the lack of a clear method of analysis. Prior research on visual hierarchy, for example, has had to limit their scope to predicting initial fixations [8]. Surveying the eye-tracking literature more broadly, the few attempts at analyzing sequence information have employed proxies, such as sectioning stimuli into regions [e.g., 1, 11], assigning values to every pixel [20], or have used vector-based methods [10]. To directly assess the notion of visual hierarchy, a novel measure is needed to analyze sequences of points in space.

Recent methodological developments establish a suitable analysis of eye movements called Fréchet distance in eye-tracking research $[6,25]$. It is a measure of similarity between two curves (i.e., trajectories or sequences of points). The more similar they are, the smaller the Fréchet distance is. Specifically, with regards to eye-tracking data, sequences of fixations are treated as points along a curve. Between two people, there are two curves that are compared using Fréchet's distance-smaller distances indicate more similar eye movements. Here we use Fréchet distance to analyze eye-tracking data while participants evaluate the design of various posters. Because Fréchet distance is a novel eye movement measure in psychology, we include the Fréchet distance of smooth pursuit stimuli to provide a baseline for our measures.

If visual hierarchy as a principle of good design is valid, more similar eye movements should bear some relationship to impressions of good design. Therefore, in accordance with this graphic design heuristic, we hypothesize that visual hierarchy (similar eye movements) is stronger when two people share a stronger joint evaluation of good design (low difference between and high average across). We test this relationship with graphic design posters.

\section{METHOD}

\subsection{Participants}

Thirty participants (22 female, 8 male) were recruited online for our study. All participants were undergraduate students (ages 18-22; mean $=19.77, S D=1.83$ ), and had normal or corrected-to-normal vision. 


\subsection{Stimuli}

\subsubsection{Visual Hierarchy}

The stimuli were collected via Google image searches for various posters. We collected 120 posters and placed them on white backgrounds, and all stimuli were standardized to 1024 by 768 pixels. The content of the posters represented a wide range of topics, e.g., concert event, grocery advertisement. The intention was to represent an ecologically valid range of aesthetics and visual hierarchies. To control for the aesthetic contribution of colors [e.g., 16], saturation was removed.

\subsubsection{Smooth Pursuit}

Our smooth pursuit stimuli consisted of videos showing a black circle moving smoothly across the screen on an invisible trajectory. The circle briefly pauses every time it changes directions. The pause is meant to solicit the recording of a specific fixation using an eye tracker. There were five different animations, each featuring a different path. Each animation lasted five seconds.

\subsection{Procedure}

\subsubsection{Visual Hierarchy}

Throughout this study, participants' eye movements were recorded using an eye tracker. Participants sat in front of a monitor with their head on a chin rest to provide stability and comfort. Each participant viewed 120 posters for five seconds each. The order of the posters was randomized for each participant. After viewing each poster, participants were prompted with the question: "How good was the design of that poster?" Participants used a keyboard to respond on a scale from 1 (not good at all) to 9 (extremely good). Once a number was selected, a fixation symbol ("+") appeared at the center of the screen. Once the participant fixated on the symbol, it was replaced by the next stimulus. Participants rated 60 stimuli in a row before being asked to take a break.

\subsubsection{Smooth Pursuit}

After participants evaluated all the posters, they were immediately shown the smooth pursuit stimuli while still having their eyes tracked. Participants were asked to follow the circle's movement with their eyes. Each animation was presented twice, and the order of the clips was random.

\subsection{Eye tracker}

We used an SR Research EyeLink 1000 Plus system to track participants' eye movements. The accuracy of this system is typically between $0.25^{\circ}$ and $0.50^{\circ}$. We tracked the left eye with a sample rate of $250 \mathrm{~Hz}$. Head position was stabilized with a chin rest placed 23 centimeters from the screen. The screen resolution was $1600 \times 1200$ at $75 \mathrm{~Hz}$. The experiment was built using SR Research Experiment Builder. We conducted a ninepoint calibration at the beginning of each experiment, and the participant navigated the experiment using the number keys and space bar on the keyboard in front of them. 


\subsection{Measures}

\subsubsection{Primary Analysis: Visual Hierarchy}

We measured the similarity of fixation sequences across participants using a metric called the Fréchet distance; a measurement of the maximum distance between two points on two different trajectories [25]. In our case, the sequence of fixations across time represents the trajectories being analyzed. The smaller the distance, the more similar the eye movements are. For each stimulus and pairwise combination of individuals viewing it, we calculated the Fréchet distance using the longitudinalData package (version 2.4.1) in $R$ (version 4.0.2). Eye movement for each image is modelled as a polygonal curve defined by the sequence of fixation points $\left(\mathrm{x}_{1}, \mathrm{y}_{1}\right)$, $\ldots,\left(x_{m}, y_{m}\right)$. Given curves $c, e:[0,1] \rightarrow R^{2}$, the Fréchet distance is formalized as

$$
\delta(c, e)=\inf _{p, q \in F} \sup _{t \in[0,1]} d(c(p(t)), e(q(t)))
$$

where $F$ is the family of continuous monotonic surjective functions $[0,1]->[0,1]$, and $d$ is Euclidean distance.

Our primary test was the significance of the relationship between Fréchet distances and two aspects of a joint impression of good design: the difference between, and the magnitude of the average across. In accordance with the visual hierarchy hypothesis, the two values together help to focus on the specific effects of positive joint impressions of good design. For example, when two participants rate a poster as 9 , the difference is 0 and the average is 9 ; the most positive joint impression. Using a mixed effects regression, we analyze the significance of the difference and magnitude of joint evaluations, along with their interaction, as predictive of Fréchet distances; in other words, whether shared impressions of good design significantly predict similar eye movements. See Figure 1 for a sample from our pilot data exemplifying the visual hierarchy hypothesis. Within our mixed effects model, variance associated with the stimuli and participant pairing were accounted for as random effects. We ran our analysis using the Ime4 package (version 1.1-21) in R. Our model using the $R$ nomenclature is as follows: FréchetDistance $\sim$ Difference*Magnitude $+(1 \mid$ pairing $)+(1 \mid$ stimulus $)$. 

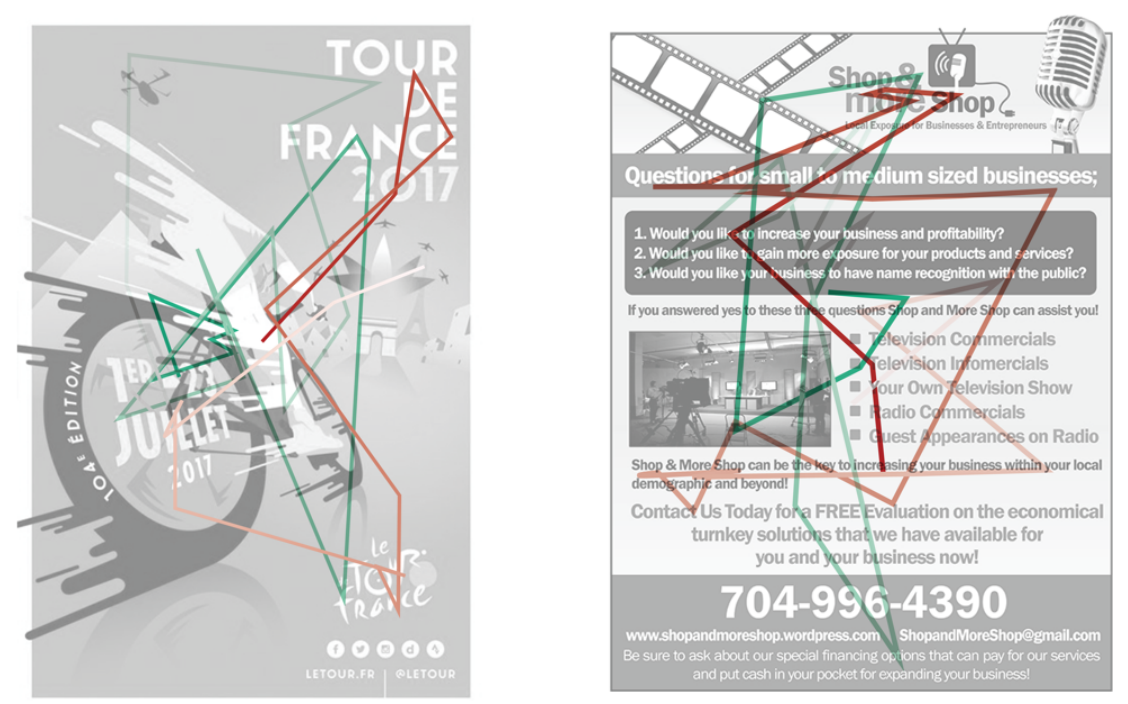

Figure 1: Eye movements recorded in the pilot study. Red and green colors represent different participants' eye movements. Darker lines represent earlier movements. The poster on the left solicited a stronger joint impression of good design and more similar eye movements (stronger visual hierarchy).

\subsubsection{Secondary Analysis: Smooth Pursuit}

A Fréchet distance of zero represents perfectly similar eye movements; however, the range of possible values does not have a finite maximum. Thus, this secondary analysis situates the values recorded during the primary poster task. If Fréchet distance is a valid measure of eye movement similarity, the smooth pursuit task should produce significantly lower values when compared to the primary task. We compare the mean values associated with both tasks using a $t$-test.

\subsection{Pre-registration}

Details regarding this experiment and its results were pre-registered <https://osf.io/dtgfu>. We conducted this study twice. After the first experiment, we found a minor flaw in the experiment, which led us to conduct the study again after fixing the flaw. We report the results of the second study. Information regarding minor discrepancies between the two studies can be found online along with our pre-registration.

\section{RESULTS}

\subsection{Primary Analysis: Visual Hierarchy}

The regression analysis shows that the interaction between joint evaluation magnitude and difference has a significant effect on the predicted Fréchet distance ( $\beta_{\text {Difference:Magnitude }}=0.99, p<0.001$ ). Fréchet distances are smaller when the difference within joint evaluations is smaller ( $\beta_{\text {Difference }}=-5.54, p<0.001$ ) relative to the magnitude of the joint evaluation ( $\beta_{\text {Magnitude }}=-3.36, p<0.001$ ), see Table 1. These results provide support for the notion of visual hierarchy; similarity in eye movements relates to joint evaluations of good design. 
Table 1: Regression for pairwise joint evaluations (magnitude and difference) and Fréchet distance.

\begin{tabular}{|c|c|c|c|c|}
\hline & $\mathrm{N}$ & $\beta$ & SE & SD \\
\hline & 30 & & & \\
\hline \multicolumn{5}{|l|}{ Fixed Effects } \\
\hline Intercept & & $364.98^{* * *}$ & 6.07 & \\
\hline Difference & & $-5.54^{\star * *}$ & 1.48 & \\
\hline Magnitude & & $-3.36^{* * *}$ & 0.68 & \\
\hline Difference:Magnitude & & $.99^{* * *}$ & 0.28 & \\
\hline \multicolumn{5}{|l|}{ Random Effects } \\
\hline Pairing & & & & 58.02 \\
\hline Stimulus & 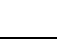 & & & 44.35 \\
\hline
\end{tabular}

To visualize the notion of visual hierarchy, we map the mean Fréchet distance with respect to average measures of both joint evaluation metrics (difference and magnitude); see Figure 2. Note that the average difference between two individuals' evaluations of a stimulus was 1.76 (median $=1$ ). Figure 2 visualizes the results of the regression and our hypothesis: the average Fréchet distance tended to be smaller as the joint evaluation magnitude increased and the constituent differences in evaluations decreased.

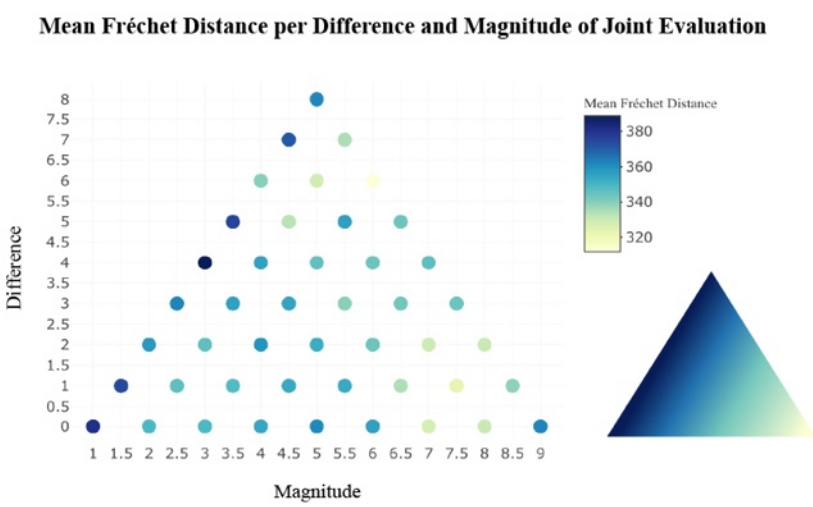

Figure 2: The mean Fréchet distances represented by color in relation to joint evaluation statistics (difference and magnitude). The triangle gradient on the lower right represents the hypothesized relationship.

\subsection{Secondary Analysis: Smooth Pursuit}

The range in Fréchet distances with regards to the poster stimuli was 1362.92 ( $\min .=64.64$, $\max .=1427.56$ ), with a mean of 347.13 . To situate these values, we tested participant's similarity in eye movement while engaging in a smooth pursuit task. In this situation, eye movements should be maximally similar and therefore the value of Fréchet distance should be smaller. The range in Fréchet distances with regards to the smooth pursuit stimuli was 1164.59 ( $\min .=83.33$, max. $=1247.92)$, with a mean of 243.23 . This mean is significantly lower than the mean of Fréchet distances with regards to the poster stimuli $(p<0.001)$; see Figure 3 . As expected, the Fréchet distances representing the smooth pursuit task approaches the lower values observed during the primary task involving posters. 
Fréchet Distances for Poster Stimuli and Smooth Pursuit Stimuli

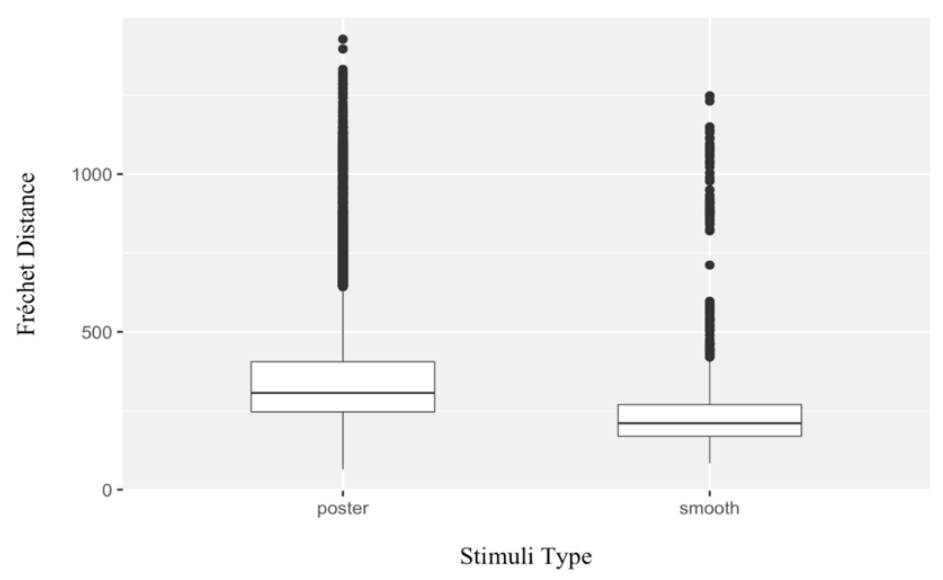

Figure 3: Boxplot showing Fréchet distances for poster stimuli and smooth pursuit stimuli. The Fréchet distance associated with the smooth pursuit stimuli provides a baseline for eye movement similarity in our method.

\section{GENERAL DISCUSSION}

Our results connect aesthetics with eye movements in the context of graphic design. Previous studies drawing this connection employed eye movements to map saliency within an image [e.g., 7, 15, 21]. We add to this literature by using a new method that considers the sequence of eye movements. Researchers have posited the potential value of analyzing sequence information to better capture the way people view a design over time [e.g., 1, 10]. By showing evidence for visual hierarchy through this method, our results fortify the idea that visual hierarchy contributes to good design.

For designers, our study represents a valuable direct test of what they proselytize today [e.g., 5]. Designers care about aesthetics; good design can make something seem more valuable [4], usable [e.g., 9, 24], and trustworthy $[19,22]$. Thus, the value of finding empirical evidence for visual hierarchy is not only theoretical, but also carries practical implications. Future research may wish to explore and empirically test the validity of techniques graphic designers posit as affecting a visual hierarchy.

For psychologists, the relationship between strong visual hierarchies and positive impressions provides evidence relating the premotor theory of attention [e.g., 2] with the fluency theory of aesthetics [e.g., 18]. It could be that posters with clear visual hierarchy have visual cues that make it easier for covert attention to work with overt attention in the planning and execution of eye movements. In other words, graphic design posters with good visual hierarchy may provide information orienting covert attention and subsequently leading eye movements to fixate on specific locations in a specific order. The cognitive ease that may come from lucid instructions about where to look next may be the reason why the presence of a strong visual hierarchy is aesthetic.

Future research should look further into the factors that may influence the relationship between visual hierarchy and aesthetics. For example, eye movement studies have found that people with art experience gaze differently at artworks [e.g., 14]. There are also likely effects based on the norms and conventions of the culture 
that the viewer is a part of [20,23]. Additionally, prior research suggests that eye movement patterns change based on the task that the viewer is engaged in [7]. While participants were specifically tasked with evaluating the aesthetics of a poster in our study, people may also engage in posters in search of specific information. It is therefore unclear how task demands may affect the relationship between visual hierarchy and impressions of good design.

This study introduces a novel method for studying aesthetics and design. This is important because aesthetics influences the way people interact with products [e.g., 4], websites [e.g., 19], and advertisements [e.g., 9]. Using Fréchet distance, we provide evidence for the notion of visual hierarchy and its link to impressions of good design. This finding gives a theoretical framework for future research endeavoring to understand aesthetics and attention.

\section{REFERENCES}

[1] Zoya Bylinskii, Michelle A. Borkin, Nam Wook Kim, Hanspeter Pfister, and Aude Oliva. 2017. Eye Fixation Metrics for Large Scale Evaluation and Comparison of Information Visualizations. In M. Burch, L. Chuang, B. Fisher, A. Schmidt, \& D. Weiskopf (Eds.), Eye Tracking and Visualization (pp. 235-255). Springer International Publishing. https://doi.org/10.1007/978-3-319-47024-5_14

[2] Soazig Casteau, and Daniel T. Smith. 2020. Covert attention beyond the range of eye-movements: Evidence for a dissociation between exogenous and endogenous orienting. Cortex, 122, 170-186. https://doi.org/10.1016/j.cortex.2018.11.007

[3] Wayne Collins, Alex Hass, Ken Jeffery, Alan Martin, Roberto Medeiros, and Steve Tomljanovic. 2015. Graphic design and print production fundamentals. BCcampus. https://opentextbc.ca/graphicdesign/

[4] Mariëlle E. H. Creusen and Jan P. L. Schoormans. 2005. The different roles of product appearance in consumer choice. Journal of Product Innovation Management, 22(1), 63-81. https://doi.org/10.1111/j.0737-6782.2005.00103.x

[5] David Dabner, Sandra Stewart, and Eric Zempol. 2004. Graphic design school: The principles and practice of graphic design (5th Edition). John Wiley \& Sons Inc.

[6] Filip Dechterenko and Jiri Lukavsky. 2018. Robustness of metrics used for scanpath comparison. Proceedings of the 2018 ACM Symposium on Eye Tracking Research \& Applications, 1-5. https://doi.org/10.1145/3204493.3204580

[7] Soussan Djamasbi, Marisa Siegel, and Tom Tullis. 2011. Visual hierarchy and viewing behavior: An eye tracking study. Proceedings of the 14th International Conference on Human-Computer Interaction, 331-340. https://doi.org/10.1007/978-3-642-21602-2 36

[8] Rebecca Grier, Philip Kortum, and James Miller. 2007. How users view web pages: An exploration of cognitive and perceptual mechanisms. In Human Computer Interaction Research in Web Design and Evaluation (pp. 22-41). IGI Global.

[9] Rebecca Hagen and Kim Golombisky. 2017. White space is not your enemy: A beginner's guide to communicating visually through graphic, web, \& multimedia design (3rd Edition). Taylor \& Francis Group, LLC.

[10] Halszka Jarodzka, Kenneth Holmqvist, and Marcus Nyström. 2010. A vector-based, multidimensional scanpath similarity measure. Proceedings of the 2010 Symposium on Eye-Tracking Research \& Applications, 211-218. https://doi.org/10.1145/1743666.1743718

[11] Olivier Le Meur, and Thierry Baccino. 2013. Methods for comparing scanpaths and saliency maps: Strengths and weaknesses. Behavior Research Methods, 45(1), 251-266. https://doi.org/10.3758/s13428-012-0226-9

[12] Ellen Lupton and Jennifer Cole Phillips. 2015. Graphic design: The new basics (2nd Edition). Princeton Architectural Press.

[13] Jakob Nielsen. 2006. F-Shaped Pattern For Reading Web Content (original eyetracking research). Nielsen Norman Group. https://www.nngroup.com/articles/f-shaped-pattern-reading-web-content-discovered/

[14] C. F. Nodine, P. J. Locher, and E. A. Krupinski. 1993. The role of formal art training on perception and aesthetic judgment of art compositions. Leonardo, 26(3), 219-227.

[15] Calvin Nodine, Claudia Mello-Thoms, Elizabeth Krupinski, and Paul Locher. 2008. Visual interest in pictorial art during an aesthetic experience. Spatial Vision, 21(1-2), 55-77. https://doi.org/10.1163/156856807782753868

[16] Stephen E. Palmer, Karen B. Schloss, and Jonathan Sammartino. 2013. Visual Aesthetics and Human Preference. Annual Review of Psychology, 64(1), 77-107. https://doi.org/10.1146/annurev-psych-120710-100504

[17] Michael I. Posner. 1980. Orienting of attention. Quarterly Journal of Experimental Psychology, 32(1), 3-25. https://doi.org/10.1080/00335558008248231

[18] Rolf Reber, Norbert Schwarz, and Piotr Winkielman. 2004. Processing Fluency and Aesthetic Pleasure: Is Beauty in the Perceiver's Processing Experience? Personality and Social Psychology Review, 8(4), 364-382. https://doi.org/10.1207/s15327957pspr0804_3

[19] David Robins, Jason Holmes, and Mary Stansbury. 2010. Consumer health information on the web: The relationship of visual design and perceptions of credibility. Journal of the American Society for Information Science and Technology, 61(1), 13-29. https://doi.org/10.1002/asi.21224

[20] Jeremiah D. Still. 2017. Web page attentional priority model. Cognition, Technology \& Work, 19(2), 363-374. 
https://doi.org/10.1007/s10111-017-0411-9

[21] Jeremiah D. Still. 2018. Web page visual hierarchy: Examining Faraday's guidelines for entry points. Computers in Human Behavior, 84, 352-359. https://doi.org/10.1016/i.chb.2018.03.014

[22] Kai-Ti Tseng and Yuan-Chi Tseng. 2014. The correlation between visual complexity and user trust in on-line shopping: Implications for design. Proceedings of the 16th International Conference on Human-Computer Interaction, 90-99. https://doi.org/10.1007/978-3-319$\underline{07227-2 \quad 10}$

[23] Marije van Amelsvoort, Jan van der Meij, Anjo Anjewierden, and Hans van der Meij. 2013. The importance of design in learning from node-link diagrams. https://doi.org/10.1007/s11251-012-9258-x

[24] Hans van der Heijden. 2003. Factors influencing the usage of websites: The case of a generic portal in The Netherlands. Information \& Management, 40(6), 541-549. https://doi.org/10.1016/S0378-7206(02)00079-4

[25] Calden Wloka, luliia Kotseruba, and John K. Tsotsos. 2018. Active fixation control to predict saccade sequences. Proceedings of the IEEE Conference on Computer Vision and Pattern Recognition, http://openaccess.thecvf.com/content_cvpr_2018/html/Wloka_Active_Fixation_Control_CVPR_2018_paper.htm 